Mitteilungen der Berliner Gesellschaft

für Psychiatrie und Neurologie e. V.

\title{
BGPN-Vorstandssitzung unter Pandemie-Bedingungen
}

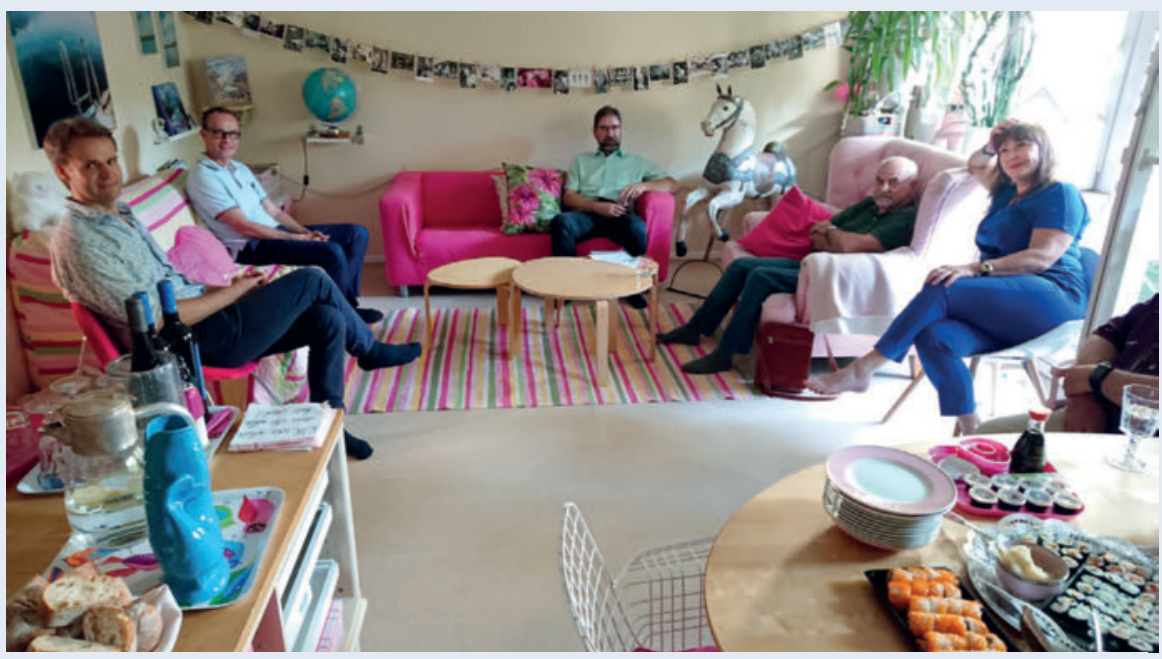

Nach coronabedingter Pause tagte der BGPN-Vorstand erstmals wieder am 5.8.2020 auf Einladung von PD Dr. Schielke. Da die Abstandsgebote strikt eingehalten wurden, zeigt das Foto nur einen kleinen Ausschnitt des Vorstands (v. I.): H. Prüß, T. Bschor, A. Hartmann, H. Gutzmann, E. Schielke. (Quelle: T. Bschor)

Aufgrund der SARS-CoV-2-bedingten Restriktionen musste die BGPN nicht nur ihre Vortragsveranstaltungen aussetzen; auch die monatlichen Vorstandssitzungen fielen zunächst aus. Dies stellte die Gesellschaft vor zunehmende Probleme, mussten doch die Anpassung des Veranstaltungskalenders an die neuen Bedingungen und das Jahres- programm 2021 besprochen werden. Per E-Mail-Korrespondenz wurde die Entscheidung getroffen, dass sich der Vorstand nicht mehr im üblichen Besprechungsraum in der Charité Campus Mitte trifft.

Am 5.8.2020 wurden die Vorstandssitzungen dann in ungewöhnlichem Rahmen wieder aufgenommen. Vorstandsmitglied PD Dr. Eva Schielke lud den Vorstand zu sich nach Hause ein, wo mit dem gebotenem Abstand zwischen den Vorstandsmitgliedern getagt werden konnte. Da die Gastgeberin auch für eine exzellente Verköstigung sorgte, nahm das Vortragsprogramm des kommenden Jahres rasch Gestalt an und wird Ihnen, sobald es fertiggestellt ist, an dieser Stelle sowie über den üblichen Mitglieder-Verteiler bekannt gegeben. Schielke sei für ihre Initiative und ihre Gastfreundschaft herzlich gedankt.

Tom Bschor, Berlin

trachtet wurde. Aufgrund seiner Sensibilität und fehlenden Rücksichtslosigkeit scheiterte er an den Anforderungen des Vaters, der dies nur mit Spott vor der gesamten Familie zu beantworten wusste. Als sich Freddy schließlich entschloss, dem Familienkonzern den Rücken zu kehren und in der aufwärtsstrebenden Flugbranche Pilot großer Passagierflugzeuge zu werden, verhöhnte ihn sein Vater als Taxifahrer der Luft. Sein jüngerer Bruder Donald Trump nutzte seine Chance und präsentierte sich beim Familienoberhaupt als der erwünschte eiskalte, ausschließlich am Geld orientierte Macher.

Die Autorin schildert weiter, wie Donald mit der zunehmenden Verantwortung im Familienkonzern durchgehend überfordert war 


\section{MARY L. TRUMP}

ZU VIEL UND NIE GENUG

Wie meine Familie

den gefährlichsten

Mann der Welt erschuf

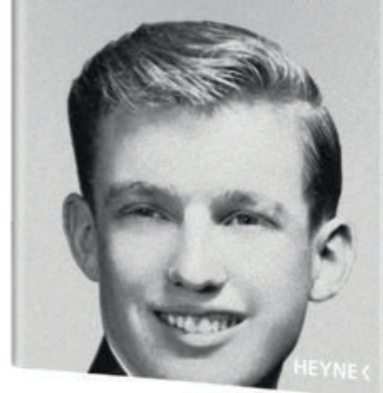

Cover des Buches von Mary L. Trump. (Quelle: Heyne Verlag)

und mit all seinen Projekten scheiterte. Kontinuierlich musste der Vater 3-stellige Millionenbeträge nachschieben. Nur mit ständigen Lügen, Prahlerei und vollkommen re- alitätsferner Selbstinszenierung konnte er beim Vater das Bild des würdigen Nachfolgers aufrechterhalten. Diese Persönlichkeitskonstanten dominieren nach Mary Trump bis heute das gesamte Handeln ihres Onkels: Unwahrheiten, fantastische Selbstüberhöhung und ein nicht stillbares Bedürfnis nach Bewunderung und Applaus.

Mary Trumps Buch bietet detaillierte Innenansichten einer hochpathologischen Familie, in dem ohne Rücksicht auf Diskretion viele - auch andere Familienmitglieder betreffende - Interna ausgeplaudert werden. Das Bedürfnis nach Klatsch und Tratsch bleibt nicht unerfüllt. Als Psychologin versteht die Autorin es, die Familiendynamik und ihren Einfluss auf die Entwicklung der Kinder plausibel nachzuzeichnen. Zugleich ist sie erkennbar keine neutrale Beobachterin. Das Bedürfnis, ihren mit 42 Jahren an einer Alkoholkrankheit und einem Herzinfarkt verstorbenen Vater zu rehabilitieren wird ebenso deutlich, wie der schon lange bestehende Bruch mit dem Rest der Familie. So berichtet sie von einem von ihr begonne- nen erbitterten Erbstreit nach dem Tod des Familienoberhaupts 1999 und teilt mit, dass sie die entscheidende Informationsquelle für einen 2018 erschienenen ausführlichen Bericht der New York Times über die Pleiten und Finanzbetrügereien von Donald Trump gewesen ist. Den Lesern fällt es freilich nicht schwer, die von Mary Trump gezeichneten Persönlichkeitszüge in der täglichen Berichterstattung nachzuvollziehen.

Tom Bschor, Berlin

IMPRESSUM

Prof. Dr. Tom Bschor

Redaktion: Dr. Anja M. Bauer

Berliner Gesellschaft für Psychiatrie und Neurologie e. V.

Schlosspark-Klinik, Abteilung für

Psychiatrie

Heubnerweg 2, 14059 Berlin

info@bgpn.de,www.bgpn.de 